\title{
Modelling the Increasing Differential Effects of the First Inter-Competition Coefficient on the Biodiversity Value; Competition between two Phytoplankton Species
}

\author{
P. Y. Igwe' ${ }^{1}$ J. U. Atsu², E.N. Ekaka-a ${ }^{3}$, and A.O. Nwaoburu ${ }^{3}$ \\ ${ }^{1}$ Department of Mathematics, Federal College of Education (Technical), Omoku, Rivers State, Nigeria. \\ ${ }^{2}$ Department of Mathematics/Statistics, Cross River University of Technology, Calabar, Nigeria. \\ ${ }^{3}$ Department of Mathematics, Rivers State University, Nkpolu,Port Harcourt, Nigeria.
}

\begin{abstract}
One of the intrinsic factors that affects the growth of two phytoplankton species is called the intercompetition coefficient. When this parameter value is decreased, the first phytoplankton specie benefit from biodiversity gain whereas the second phytoplankton specie is vulnerable to biodiversity loss. In contrast, when the same parameter value is increased from the value of 0.0525 to 0.099 the first phytoplankton specie dominantly suffers from a biodiversity loss whereas the second phytoplankton specie benefits from a biodiversity gain. The novel results that we have obtained have not been seen elsewhere but compliments our current contribution to knowledge in this challenging interdisciplinary research; these full results are presented and discussed quantitatively.
\end{abstract}

Keywords- Differential effect, inter-competition coefficient, phytoplankton specie, biodiversity richness, continuous differential equation.

\section{INTRODUCTION}

Heterogeneity of species is a very important factor in biodiversity richness. Phytoplankton play a very crucial role in ocean ecology [Saha and Bandyopadhyay 2009].

While some species of phytoplankton are known to produce toxins which can contaminate seafood, others are high producers of biomass which in high concentrations can cause mortalities of marine life. However, the toxin producing phytoplankton are known to play very significant roles in the growth of zooplankton [Bandyopadhyay et al 2008]. Atsu \& Ekaka-a (2017) have shown that as fractional order dimension increases from 0.1 to 0.75 , there is a reduction in specie depletion. The implication is that an increased fractional order dimension results in a biodiversity gain.

\section{MATERIALS AND METHODS}

We have considered the following Lotka-Volterra model equations of competition indexed by a system of continuous non-linear first order ordinary differential equations:

$$
\begin{aligned}
& \frac{d N_{1}(t)}{d t}=N_{1}(t)\left[\alpha_{1}-\beta_{1} N_{1}(t)-\gamma_{1} N_{2}(t)\right] \\
& \frac{d N_{2}(t)}{d t}=N_{2}(t)\left[\alpha_{2}-\beta_{2} N_{2}(t)-\gamma_{2} N_{1}(t)\right]
\end{aligned}
$$

Here, the initial conditions are defined by $N_{1}(0)=N_{10} \geq 0$ and $N_{2}(0)=N_{20} \geq 0$, whereas $N_{1}(t)$ and $N_{2}(t)$ specify the densities of the two phytoplankton species (measured as the number of cells per litter). For the purpose of this formulation, $\alpha_{1}$ and $\alpha_{2}$ specify the cell proliferation rate per day; $\beta_{1}$ and $\beta_{2}$ specify the rate of intra-specific competition terms for the first and second species; $\gamma_{1}$ and $\gamma_{2}$ specify the rate of inter-specific competition. The units of $\alpha_{1}, \alpha_{2}, \beta_{1}$, $\beta_{2}, \gamma_{1}$ and $\gamma_{2}$ are per day per cell and day is the unit of time. Following the parameter values as proposed by Bandyopadhyaya, et al ,2008, where $\alpha_{1}=2, \alpha_{2}=1, \beta_{1}=$ $0.07, \beta_{2}=0.08, \gamma_{1}=0.05, \gamma_{2}=0.015$.

The method is hereby stated step by step as follows:

Step 1: Consider a scenario where $N_{1}$ (old) is the predicted biomass $\left[N_{1}\right.$ (old) is the population density of the first phytoplankton species otherwise called the biomass of the first phytoplankton species when all other parameter values are fixed at time $t$.

Step 2: Replace $N_{1}$ (old) with $N_{1}$ (new) due to a variation of the inter-competition coefficient $\gamma_{1}$. 
Step 3: If $N_{1}$ (new) is strictly less than $N_{1}$ (old), it indicates that the variation of $\gamma_{1}$ has predicted a depletion which mimics biodiversity loss. In this scenario, the appropriate mathematically formula for the quantification of biodiversity loss is defined as follows:

$\mathrm{BL}(\%)=100\left[\frac{N_{1}(\text { old })-N_{1}(\text { new })}{N_{1}(\text { old })}\right]$

Step 4: If $N_{1}$ (new) is strictly greater than $N_{1}$ (old), due to the variation of $\gamma_{1}$, then a biodiversity gain has occurred which can be similarly defined as follows:

$$
\mathrm{BG}(\%)=100 \quad\left[1-\frac{\mathrm{N}_{1}(\mathrm{new})}{\mathrm{N}_{1(\mathrm{old})}}\right]
$$

\section{RESULTS}

On the application of the above mentioned methods, we have obtained the following empirical results that we have not seen elsewhere.

Table.1: Evaluating the extent of biodiversity for $\gamma_{1}=$ 0.049 with experimental time of 10 years using ODE 45

\begin{tabular}{rrrrrrr}
\hline $\boldsymbol{N}_{\mathbf{1}}$ & $\boldsymbol{N}_{\mathbf{1} \boldsymbol{m}} \mathbf{B G}(\%)$ & $\boldsymbol{N}_{\mathbf{2}} \boldsymbol{N}_{\mathbf{2 m}} \mathbf{B L}(\boldsymbol{\%})$ \\
\hline 4.0000 & 4.0000 & 0 & 10.0000 & 10.0000 & 0 \\
10.6819 & 10.7674 & 0.8010 .6094 & 10.6048 & 0.04 & \\
17.3033 & 17.4700 & 0.9610 .1236 & 10.1074 & 0.16 & \\
20.5151 & 20.6862 & 0.839 .4452 & 9.4213 & 0.25 & \\
21.7063 & 21.8641 & 0.738 .9593 & 8.9328 & 0.30 & \\
22.1823 & 22.3306 & 0.67 & 8.6637 & 8.6368 & 0.31 & \\
22.4060 & 22.5491 & 0.648 .4908 & 8.4641 & 0.31 & \\
22.5238 & 22.6639 & 0.628 .3900 & 8.3637 & 0.31 & \\
22.5896 & 22.7279 & 0.618 .3312 & 8.3051 & 0.31 & \\
22.6273 & 22.7645 & 0.618 .2967 & 8.2708 & 0.31 & \\
\hline
\end{tabular}

Table.2: Evaluating the extent of biodiversity for $\gamma_{1}=$ 0.0525 with experimental time of 10 years using ODE 45

\begin{tabular}{ccccccc}
\multicolumn{7}{c}{ numerical } \\
\hline $\boldsymbol{N}_{\mathbf{1}}$ & $\boldsymbol{N}_{\mathbf{1} \boldsymbol{m}} \boldsymbol{B L}(\%)$ & $\boldsymbol{N}_{\mathbf{2}} \boldsymbol{N}_{\mathbf{2 m}} \mathbf{B G}(\boldsymbol{\%})$ \\
\hline 4.0000 & 4.0000 & 0 & 10.0000 & 10.0000 & 0 \\
10.6819 & 10.4703 & 1.98 & 10.6094 & 10.6206 & 0.11 \\
17.3033 & 16.8882 & 2.4010 .1236 & 10.1638 & 0.40 \\
20.5151 & 20.0849 & 2.109 .4452 & 9.5052 & 0.64 \\
21.7063 & 21.3077 & 1.848 .9593 & 9.0259 & 0.74 \\
22.1823 & 21.8070 & 1.698 .6637 & 8.7316 & 0.78 \\
22.4060 & 22.0442 & 1.628 .4908 & 8.5582 & 0.79 \\
22.5238 & 22.1696 & 1.578 .3900 & 8.4567 & 0.79 \\
22.5896 & 22.2399 & 1.558 .3312 & 8.3971 & 0.79 \\
22.6273 & 22.2803 & 1.538 .2967 & 8.3621 & 0.79
\end{tabular}

Table.3: Evaluating the extent of biodiversity for $\gamma_{1}=$ 0.055 with experimental time of 10 years using ODE 45

\begin{tabular}{rrrrrr}
\hline $\boldsymbol{N}_{\mathbf{1}}$ & $\boldsymbol{N}_{\mathbf{1} \boldsymbol{m}} \mathbf{B L}(\%)$ & $\boldsymbol{N}_{\mathbf{2}} \boldsymbol{N}_{\mathbf{2 m}} \mathbf{B G}(\boldsymbol{\%})$ \\
\hline 4.0000 & 4.0000 & 0 & 10.0000 & 10.0000 & 0 \\
10.6819 & 10.2618 & 3.9310 .6094 & 10.6317 & 0.21 \\
17.3033 & 16.4754 & 4.7910 .1236 & 10.2036 & 0.79 \\
20.5151 & 19.6507 & 4.219 .4452 & 9.5653 & 1.27 \\
21.7063 & 20.9031 & 3.708 .9593 & 9.0933 & 1.50 \\
22.1823 & 21.4256 & 3.418 .6637 & 8.8004 & 1.58 \\
22.4060 & 21.6763 & 3.268 .4908 & 8.6266 & 1.60 \\
22.5238 & 21.8095 & 3.178 .3900 & 8.5244 & 1.60 \\
22.5896 & 21.8844 & 3.128 .3312 & 8.4642 & 1.60 \\
22.6273 & 21.9277 & 3.098 .2967 & 8.4286 & 1.59 \\
\hline
\end{tabular}

Table.4: Evaluating the extent of biodiversity for $\gamma_{1}=$ 0.0575 with experimental time of 10 years using ODE 45

\begin{tabular}{ccccccc}
\hline $\boldsymbol{N}_{\mathbf{1}} \boldsymbol{N}_{\mathbf{1} \boldsymbol{m}} \mathbf{B L}(\%)$ & $\boldsymbol{N}_{\mathbf{2}} \boldsymbol{N}_{\mathbf{2} \boldsymbol{m}} \mathbf{B G}(\boldsymbol{\%})$ \\
\hline 4.0000 & 4.0000 & 0 & 10.0000 & 10.0000 & 0 \\
10.6819 & 10.0566 & 5.8510 .6094 & 10.6427 & 0.31 & \\
17.3033 & 16.0652 & 7.1610 .1236 & 10.2432 & 1.18 \\
20.5151 & 19.2130 & 6.35 & 9.4452 & 9.6254 & 1.91 & \\
21.7063 & 20.4927 & 5.59 & 8.9593 & 9.1613 & 2.26 & \\
22.1823 & 21.0378 & 5.16 & 8.6637 & 8.8701 & 2.38 \\
22.4060 & 21.3021 & 4.93 & 8.4908 & 8.6961 & 2.41 & \\
22.5238 & 21.4434 & 4.80 & 8.3900 & 8.5932 & 2.42 \\
22.5896 & 21.5231 & 4.72 & 8.3312 & 8.5323 & 2.41 & \\
22.6273 & 21.5693 & 4.68 & 8.2967 & 8.4962 & 2.40 & \\
\hline
\end{tabular}

Table.5: Evaluating the extent of biodiversity for $\gamma_{1}=0.06$ with experimental time of 10 years using ODE 45

\begin{tabular}{rccccc}
\hline $\boldsymbol{N}_{\mathbf{1}}$ & $\boldsymbol{N}_{\mathbf{1} \boldsymbol{m}} \mathbf{B L}(\%)$ & $\boldsymbol{N}_{\mathbf{2}} \boldsymbol{N}_{\mathbf{2} \boldsymbol{m}} \mathbf{B G}(\boldsymbol{\%})$ \\
\hline 4.0000 & 4.0000 & 0 & 10.0000 & 10.0000 & 0 \\
10.6819 & 9.8545 & 7.75 & 10.6094 & 10.6536 & 0.42 \\
17.3033 & 15.6578 & 9.51 & 10.1236 & 10.2825 & 1.57 \\
20.5151 & 18.7722 & 8.50 & 9.4452 & 9.6857 & 2.55 \\
21.7063 & 20.0764 & 7.51 & 8.9593 & 9.2299 & 3.02 \\
22.1823 & 20.6436 & 6.94 & 8.6637 & 8.9408 & 3.20 \\
22.4060 & 20.9216 & 6.63 & 8.4908 & 8.7666 & 3.25 \\
22.5238 & 21.0711 & 6.45 & 8.3900 & 8.6631 & 3.25 \\
22.5896 & 21.1558 & 6.35 & 8.3312 & 8.6015 & 3.25 \\
22.6273 & 21.2050 & 6.29 & 8.2967 & 8.5649 & 3.23 \\
\hline
\end{tabular}

Table.6: Evaluating the extent of biodiversity for $\gamma_{1}=$ 0.095 with experimental time of 10 years using ODE 45

\begin{tabular}{rrrrrr}
\hline $\boldsymbol{N}_{\mathbf{1}}$ & $\boldsymbol{N}_{\mathbf{1} \boldsymbol{m}} \mathrm{BL}(\%)$ & \multicolumn{1}{c}{$\boldsymbol{N}_{\mathbf{2}} \boldsymbol{N}_{\mathbf{2} \boldsymbol{m}} \mathbf{B G}(\boldsymbol{\%})$} \\
\hline 4.0000 & 4.0000 & 0 & 10.0000 & 10.0000 & 0 \\
10.6819 & 7.3483 & 31.21 & 10.6094 & 10.7915 & 1.72
\end{tabular}




\begin{tabular}{llllll}
17.3033 & 10.3835 & 39.99 & 10.1236 & 10.7873 & 6.56 \\
20.5151 & 12.4577 & 39.28 & 9.4452 & 10.5173 & 11.35 \\
21.7063 & 13.7002 & 36.88 & 8.9593 & 10.2380 & 14.27 \\
22.1823 & 14.4254 & 34.97 & 8.6637 & 10.0218 & 15.68 \\
22.4060 & 14.8564 & 33.70 & 8.4908 & 9.8706 & 16.25 \\
22.5238 & 15.1193 & 32.87 & 8.3900 & 9.7695 & 16.44 \\
22.5896 & 15.2828 & 32.35 & 8.3312 & 9.7030 & 16.47 \\
22.6273 & 15.3860 & 32.00 & 8.2967 & 9.6598 & 16.43 \\
\hline
\end{tabular}

Table.7: Evaluating the extent of biodiversity for $\gamma_{1}=$ 0.0975 with experimental time of 10 years using ODE 45

\begin{tabular}{rrrrrc}
\hline $\boldsymbol{N}_{\mathbf{1}}$ & $\boldsymbol{N}_{\mathbf{1} \boldsymbol{m}} \mathrm{BL}(\%)$ & $\boldsymbol{N}_{\mathbf{2}} \boldsymbol{N}_{\mathbf{2 m}} \mathbf{B G}(\boldsymbol{\%})$ \\
\hline 4.0000 & 4.0000 & 0 & 10.0000 & 10.0000 & 0 \\
10.6819 & 7.1914 & 32.6810 .6094 & 10.8004 & 1.80 \\
17.3033 & 10.0462 & 41.94 & 10.1236 & 10.8196 & 6.88 \\
20.5151 & 12.0143 & 41.44 & 9.4452 & 10.5739 & 11.95 \\
21.7063 & 13.2174 & 39.11 & 8.9593 & 10.3112 & 15.09 \\
22.1823 & 13.9344 & 37.18 & 8.6637 & 10.1041 & 16.63 \\
22.4060 & 14.3678 & 35.88 & 8.4908 & 9.9574 & 17.27 \\
22.5238 & 14.6356 & 35.02 & 8.3900 & 9.8580 & 17.50 \\
22.5896 & 14.8041 & 34.47 & 8.3312 & 9.7920 & 17.54 \\
22.6273 & 14.9113 & 34.10 & 8.2967 & 9.7487 & 17.50 \\
\hline
\end{tabular}

Table.8: Evaluating the extent of biodiversity for $\gamma_{1}=$ 0.099 with experimental time of 10 years using ODE 45

\begin{tabular}{cccccc}
\hline $\boldsymbol{N}_{\mathbf{1}} \boldsymbol{N}_{\mathbf{1} \boldsymbol{m}} \mathbf{B L}(\%)$ & \multicolumn{7}{c}{$\boldsymbol{N}_{\mathbf{2}} \boldsymbol{N}_{\mathbf{2 m}} \mathbf{B G}(\boldsymbol{\%})$} \\
\hline 4.0000 & 4.0000 & 0 & 10.0000 & 10.0000 & 0 \\
10.6819 & 7.0987 & 33.5510 .6094 & 10.8057 & 1.85 \\
17.3033 & 9.8468 & 43.09 & 10.1236 & 10.8388 & 7.07 \\
20.5151 & 11.7501 & 42.73 & 9.4452 & 10.6075 & 12.31 \\
21.7063 & 12.9272 & 40.45 & 8.9593 & 10.3550 & 15.58 \\
22.1823 & 13.6374 & 38.52 & 8.6637 & 10.1537 & 17.20 \\
22.4060 & 14.0713 & 37.20 & 8.4908 & 10.0099 & 17.89 \\
22.5238 & 14.3415 & 36.33 & 8.3900 & 9.9117 & 18.14 \\
22.5896 & 14.5127 & 35.76 & 8.3312 & 9.8461 & 18.18 \\
22.6273 & 14.6223 & 35.38 & 8.2967 & 9.8028 & 18.15 \\
\hline
\end{tabular}

\section{DISCUSSION OF RESULTS.}

With an inter-competition coefficient value of $\gamma_{1}=0.049$ and an experimental time of ten (10) years, the biodiversity gain percentage value has maintained a maximum value of 0.96 and biodiversity loss maximum percentage value of 0.31 from the sixth month. As $\gamma_{1}$ increases to 0.0525 , the maximum biodiversity gain percentage value is 2.40 occurs at the second month while the maximum biodiversity loss percentage value of 0.79 starts occurring from the seventh month. At an $\gamma_{1}$ value of 0.055 , the maximum biodiversity gain percentage value is 4.79 and biodiversity loss percentage value is 1.60 . When the $\gamma_{1}$ value is 0.0575 , the maximum biodiversity gain percentage value is 7.16 while the maximum biodiversity loss percentage value is 2.42 . An $\gamma_{1}$ value of 0.06 results in a maximum biodiversity gain percentage value of 9.51 at the second month while the maximum biodiversity loss percentage value is 3.25 occurring at the seventh and eighth months. When $\gamma_{1}$ is 0.095 , the maximum biodiversity gain percentage value is 39.99 and maximum biodiversity loss percentage value is 16.47. At $\gamma_{1}=0.0975$, the maximum biodiversity gain value is 41.94 while maximum biodiversity loss value is 17.54 and when $\gamma_{1}=0.099$, maximum biodiversity gain percentage value is 43.09 and maximum biodiversity loss percentage value is maintained at 18.18 . Predominantly a biodiversity gain is predicted at the second month while a biodiversity loss is predicted from the seventh month and higher.

\section{CONCLUSION}

The MATLAB ODE45 numerical scheme has been used to predict biodiversity gain and biodiversity loss resulting from inter-competition between two phytoplankton species at increasing inter-competition coefficient levels. A relatively higher inter-competition coefficient would result in a relatively lower biodiversity gain.

\section{REFERENCES}

[1] Anderson D.M. (1989). Toxic algae blooms and red tides: a global perspective, in: T. Okaichi, D.M. Anderson, T. Nemoto (Eds.), Red Tides: Biology, Environ. Sci. Toxicol., Elsevier, New York, pp. 1121.

[2] Atsu, J. U. \& Ekaka-a, E. N.(2017). Modelling intervention with respect to biodiversity loss: A case study of forest resource biomass undergoing changing length of growing season. International Journal of Engineering, Management and Science. 3(9).

[3] Bandyopadhyaya M., Saha T., Pal R. (2008). Deterministic and Stochastic analysis of a delayed allelopathic phytoplankton model within fluctuating environment, Elsevier, 2:958-970.

[4] Bandyopadhyaya M. (2006). Dynamical analysis of a allelopathic phytoplankton model, J. Biol. Sys. 14: 205-218.

[5] Chattopadhyay J., Sarkar R.R., Mondal S. (2002). Toxin-producing phytoplankton may act as a biological control for planktonic blooms-field study and mathematical modeling, J. Theor. Biol. 215: 333344. 
[6] Chattopadhyay J. (1996). Effects of toxic substances on a two-species competitive system, Ecol. Model. 84: 287-289.

[7] Common, M., Perrings, C. (1992). Towards an ecological economics of sustainability. Ecol. Economics 6, 7-34.

[8] Duinker J., Wefer G (1994). Das $\mathrm{CO}_{2}$ und die rolle des ozeans, Naturwis sens chahten, 81:237-242.

[9] Ekaka-a E.N (2009). Computational and mathematical modelling of plant species interactions in a harsh climate. Ph.D Thesis, Department of Mathematics, The University of Liverpool and The University of Chester, United Kingdom, 2009.

[10] Hallegraeff G.M. (1993). A review of harmful algae blooms and the apparent global increase, Phycologia 32:79-99.

[11] Hernándes-Bermejo, B., Fairén, V., (1995). LotkaVolterra representation of general nonlinear systems.Math. Biosci. 140: 1-32.

[12] Loreau, M., (2000). Biodiversity and ecosystem functioning: recent theoretical advances. Oicos 91(1), 3-17.

[13] May R.M. (2001). Stability and Complexity in Model Ecosystems, Princeton University Press, New Jercy.

[14] Maynard-Smith J. (1974), Models in Ecology, Cambridge University Press, Cambridge.

[15] Nisbet R.M., Gurney W.S.C. (1982). Modelling Fluctuating Populations, Wiley Interscience, New York.

[16] Perrings, C., (1995). Ecological resilience in the sustainability of economic development. Economie Appliquée 48(2), 121-142.

[17] Pykh Yu, A. (2002). Lyapunov functions as a measure of biodiversity:theoretical background, Ecological Indicators 2: 123-133.

[18] Rice E. (1984). Allelopathy, Academic Press, New York.

[19] Saha, T. \& Bandyopadhyay, M. (2009). Dynamical analysis of toxin producing phytoplankton interactions. Nonlinear Analysis, Real World Applications 10: 314-332.

[20] Sarkar R.R., Chattopadhyay J. (2003). The role of environmental stochasticity in a toxic phytoplanktonnon-toxic phytoplankton-zooplankton system, Environmetrics, 14: 775-792.

[21] Smayda T. (1990). Novel and nuisance phytoplankton blooms in the sea: Evidance for a global epidemic, In: E. Graneli, B. Sundstrom, L. Edler, D.M. Anderson
(Eds.), Toxic Marine Phytoplankton, Elsevier, New York , pp. 29-40.

[22] Solé J., García-Ladona E., Ruardij P., Estrada M. (2005). Modelling allelopathy among marine algae, Ecol. Model. 183:373-384.

[23] Tapaswi P.K., Mukhopadhyay A. (1999). Effects of environmental fluctuation on plankton allelopathy, J. Math. Biol. 39: 39-58 